\title{
Prevalence of Anemia in Chinese Children and Adolescents and Its Associated Factors
}

\author{
Jinghuan Wu®, Yichun Hu®, Min Li, Jing Chen, Deqiao Mao, Weidong Li, Rui Wang, \\ Yanhua Yang, Jianhua Piao, Lichen Yang * and Xiaoguang Yang *
}

Key Laboratory of Trace Element Nutrition of National Health Commission of the People's Republic of China, Department of Trace Element Nutrition, National Institute for Nutrition and Health, Chinese Center for Disease Control and Prevention, No. 29 Nan Wei Road, Xicheng District, Beijing 100050, China; jhwu2012@163.com (J.W.); huyc@ninh.chinacdc.cn (Y.H.); limin@ninh.chinacdc.cn (M.L.); chenjing@ninh.chinacdc.cn (J.C.); maodq@ninh.chinacdc.cn (D.M.); liwd@ninh.chinacdc.cn (W.L.); wangrui@ninh.chinacdc.cn (R.W.); yangyh@ninh.chinacdc.cn (Y.Y.); piao_jianhua@sohu.com (J.P.)

* Correspondence: yanglichen28@126.com (L.Y.); xgyangcdc@vip.sina.com (X.Y.); Tel.: +86-10-66237176 (L.Y.); +86-10-66237273 (X.Y.)

Received: 27 March 2019; Accepted: 17 April 2019; Published: 19 April 2019

\begin{abstract}
In this study, we assessed the hemoglobin levels and anemia status of Chinese children and adolescents from the Chinese National Nutrition and Health Survey (CNNHS) in 2010-2012 and analyzed the factors associated with anemia. The hemoglobin concentration and prevalence of anemia for children and adolescents aged 6-17 years from both CNNHS 2010-2012 and CNNHS 2002 were analyzed. Multi-variable logistic regression analysis was used to assess the factors associated with anemia. The mean hemoglobin concentration increased among Chinese children and adolescents, from $135.2 \pm 13.9 \mathrm{~g} / \mathrm{L}$ in CNNHS 2002 to $141.2 \pm 15.8 \mathrm{~g} / \mathrm{L}$ in CNNHS 2010-2012, with the prevalence of anemia decreasing from $12.6 \%$ to $6.6 \%(p<0.0001)$. Anemia was specifically related to girls $(p<0.0001)$; children aged $6-8$ years $(p=0.0175), 12-14$ years $(p=0.0007)$, and $15-17$ years $(p<0.0001)$; ordinary rural areas $(p=0.0009)$ and poor rural areas $(p<0.0001)$; spring $(p<0.0001)$, autumn $(p<0.0001)$, and winter $(p<0.0001)$; underweight individuals $(p<0.0001)$; and an annual average income per capita of less than 20,000 RMB $(p<0.0001)$. The prevalence of anemia in Chinese children and adolescents has improved significantly in comparison to 10 years prior; however, it remains a public health problem in this population. Further research is required to understand the determinants of iron status, which could then lead to strategies to alleviate iron deficiency for Chinese children and adolescents, especially for girls, those living in rural areas, underweight individuals, and those with a low family income.
\end{abstract}

Keywords: anemia; hemoglobin; Chinese children and adolescents; nutrition survey

\section{Introduction}

Anemia refers to a condition in which the number of red blood cells or their oxygen-carrying capacity is insufficient to meet physiological needs [1,2]. Anemia adversely impacts health and social economic development; children and women are particularly vulnerable [1,2]. In children, anemia may detrimentally affect cognitive development and physical growth from infancy to adolescence [3] and is associated with increased morbidity [4]. Anemia continues to be an important public health concern globally. The world wide anemia prevalence in 2010 was $32.9 \%$ in children and adults [5]; it is considerably more prevalent in developing than in developed countries [6].

Iron deficiency is estimated to be the most common cause of anemia worldwide [7]. In 2013, 1.2 billion people suffered from iron-deficiency anemia [8]. Other causes of anemia include parasitic 
infections, other nutritional deficiencies, such as folate, vitamin $\mathrm{B}_{12}$, and vitamin $\mathrm{A}$ deficiencies, chronic inflammation, and inherited disorders [7]. Hemoglobin levels can also vary greatly according to age, sex, altitude, smoking, and pregnancy status [9].

Much research has been reported on the prevalence of anemia worldwide; much of it focused on children under five years old (0-59 months) or pregnant women [6]; however, anemia data from children aged above five years and adolescents are limited. In China, although a few studies have investigated hemoglobin concentration and the prevalence of anemia in children and adolescents, they were based on relatively small sample sizes or regional compositions with specific populations, which made the studies unrepresentative [10-12].

The CNNHS has been administered every 10 years since 1982 to assess the nutritional status of Chinese citizens, and anemia is one of the important focuses of the survey. Hemoglobin concentration is used to evaluate anemia status in the CNNHS. According to the 2002 CNNHS, the prevalence of anemia was $20.1 \%$ for Chinese residents [13]. Since the late 2002s, the Chinese economy has undergone rapid development [14]; meanwhile, people's dietary patterns have changed drastically [15]. In 2010-2012, China carried out its fourth National Nutrition and Health Survey. Using data from CNNHS 2002 and CNNHS 2010-2012, we aimed to (1) evaluate the hemoglobin concentration and the prevalence of anemia in children and adolescents aged 6-17 years, (2) compare the hemoglobin concentration and the prevalence of anemia among this population in 2002 and 2012, and (3) investigate the potential factors associated with anemia.

\section{Materials and Methods}

\subsection{Study Design and Participants}

Data of children and adolescents aged 6-17 years were obtained from CNNHS 2010-2012, which was the same survey as that reported in Li et al. [16] and Hu et al. [17]. This survey was a national, representative, and cross-sectional study administered to assess the health and nutrition status of Chinese citizens. It covered 31 provinces, autonomous regions, and municipalities directly under the central government throughout China (with the exceptions of Taiwan, Hong Kong, and Macao). A random stratified multistage cluster sampling design was used to recruit the participants of this survey. There were four strata in CNNHS 2010-2012: Large cities, small and medium-sized cities, ordinary rural areas, and poor rural areas, according to their economic characteristics and social development. Large cities included provincial capitals and municipalities with a population of more than one million. Small and medium-sized cities were defined as downtown areas that excluded the large cities. Poor rural areas were key poverty-stricken counties that were designated according to the 2001-2010 National Rural Poverty Alleviation and Development Program [18]. Ordinary rural areas were counties that were excluded from the key poverty counties. According to the population proportion, in the first stage of sampling, 34 large cities, 41 small and medium-sized cities, 45 rural areas, and 30 poor rural areas were selected as investigation sites (150 in total). The second stage involved six village committees or neighborhood committees from each investigation site. The last stage included 75 households from each selected village or neighborhood committee [19].

Data were also obtained from CNNHS 2002, which used the same design as CNNHS 2010-2012. In this survey, all counties (districts) and administrative units (including counties, county-level cities and districts) from 31 provinces in mainland China were divided into six categories: Two types of cities (large cities and small and medium-sized cities) and four types of rural areas (classes one, two, three, and four). This CNNHS included a total of 132 investigation sites in 2002. The second stage was the same as CNNHS 2010-2012. Finally, 90 households from each selected village or neighborhood committee were selected. 


\subsection{Ethical Approval}

The survey was conducted according to the Declaration of Helsinki guidelines. All procedures for the survey involving human subjects were ethically approved by the Ethics Committee of the Institute for Nutrition and Health, Chinese Center for Disease Control and Prevention (China CDC) with the file number of 2013-018. All participants in this survey signed an informed consent form. As to the children and adolescents under 18 years old, informed written consent was obtained from the participants' parents prior to the start of the study.

\subsection{Blood Sample and Hemoglobin Measurement}

The National Institute for Nutrition and Health, China CDC established a national project workgroup to develop a unified survey and questionnaire and conducted the survey using unified methods and materials. Hemoglobin levels were measured in the CDC laboratory in the district (county) of the survey area using the cyanmethemoglobin method, which is the most reliable laboratory method for the quantitative determination of hemoglobin and serves as a reference for the comparison and standardization of other methods [7]. This method is recommended by the International Committee for Standardization in Hematology [20]. Laboratory operations staff participated in the uniform national team training and examination. After becoming qualified, they could participate in the hemoglobin measurement.

After an overnight fast of at least $10 \mathrm{~h}$, peripheral whole venous blood samples were collected in ethylene diamine tetra-acetic acid (EDTA) tubes; then, a $10 \mu \mathrm{L}$ anticoagulant whole blood sample in a $10 \mu \mathrm{L}$ quantitative capillary tube (Drummond Scientific Company, Broomall, PA, USA) was collected specifically for the hemoglobin test. Each blood sample had two parallel determinations. The intra- and inter-assay coefficients of variation (CVs) in the analytical methods were $1.03-2.91 \%$ and $2.04-3.70 \%$, respectively. Blind samples (high- and low-value hemoglobin) were measured and qualified before proceeding with fieldwork. After the official commencement of fieldwork, one quality control analysis was tested in every 30 samples.

\subsection{Criteria of Anemia}

Normal hemoglobin distributions vary with sex, age, altitude, and, in women, pregnancy and menstrual status. Therefore, the correct interpretation of hemoglobin requires the consideration of modulating factors when selecting appropriate cut-off points. The age-specific cut-off points for hemoglobin at sea level are as follows: (1) For children aged under five years and pregnant women: $\geq 110 \mathrm{~g} / \mathrm{L}$ normal, $<110 \mathrm{~g} / \mathrm{L}$ anemia; (2) for children aged 5-11 years: $\geq 115 \mathrm{~g} / \mathrm{L}$ normal, $<115 \mathrm{~g} / \mathrm{L}$ anemia; (3) for children aged 12-14 years and non-pregnant women above 15 years old: $\geq 120 \mathrm{~g} / \mathrm{L}$ normal, $<120 \mathrm{~g} / \mathrm{L}$ anemia; and (4) for men above 15 years of age: $\geq 130 \mathrm{~g} / \mathrm{L}$ normal, $<130 \mathrm{~g} / \mathrm{L}$ anemia [7]. Hemoglobin levels at various altitudes were calculated according to the recommendations of the World Health Organization (WHO) [7].

\subsection{Variables}

All information concerning participants, including their age, sex, ethnicity, latitude, and family income was collected from each investigation site and recorded into the unified CNNHS System Platform. An anthropometric measurement for the participants was recorded by trained staff. Body mass index (BMI) was calculated as weight $(\mathrm{kg})$ divided by height $(\mathrm{m})$ squared, and its classification was based on the BMI z-score for individuals aged 6-17 years [21]. The season was recorded according to the month of blood sample collection. The latitude was defined according to the boundaries of China's Qinling Mountains and Huaihe River, which are recognized as the boundaries that divide the north and the south of China. 


\subsection{Data Check}

The hemoglobin level data of each monitoring site were recorded into the unified software. The unified standard of the data check principle was mainly achieved in the following aspects: (1) Connecting the hemoglobin database to the basic information base to check for duplicate information; (2) checking whether the difference in the hemoglobin values of parallel samples was less than $20 \%$; and (3) according to the absorbance value of the spectrophotometer, calculating the hemoglobin level and then comparing it with reported values. Any problematic records were rejected and returned to the monitoring site for re-inspection.

\subsection{Statistical Analysis}

The population figures released by the National Bureau of Statistics of China in 2009 were used as a standard population [22]. Hemoglobin concentration and anemia rate analyses were adjusted for sample weights and the clustered survey design [19]. All participants in this study were divided into different sub-groups such as age group, region type, ethnicity, etc., according to different hypothesized predictors for anemia status. Hemoglobin levels were expressed as the mean \pm standard deviation (SD) and were compared using the Kruskal-Wallis test. Frequencies were presented as percentages (\%) and 95\% confidence intervals (CIs). The rates of anemia were compared by the Rao-Scott test. The Bonferroni test was applied to conduct post-hoc analysis. Multivariable logistic regression analysis (proc surveylogistic) was used to analyze the relationship between anemia and possible predictors (e.g., age, sex, region type, BMI, season, family incomes). The odds ratio (OR) and 95\% CIs were determined using multivariable logistic regression models. All the statistical analyses were conducted by Statistical Analysis Systems 9.4 software (SAS Institute Inc., Cary, NC, USA). A two-tailed $p$-value $<0.05$ was considered statistically significant.

\section{Results}

\subsection{Participant Characteristics}

The distribution of children and adolescents from this survey according to age group, sex, region type, ethnicity, latitude, season, BMI, and income level is presented in Table 1 . There were 33,015 subjects that had their hemoglobin measured, including 16,721 boys (53.2\%) and 16,294 girls (46.8\%). The distribution of participants was similar in terms of region type, ethnicity, latitude, season, and income level. There was a significant difference in the distribution of children and adolescents in terms of their age group and BMI classification $(p<0.05)$. Post-hoc analysis showed that sex distribution in children aged 6-8 years was significantly different from those aged 9-11 years $(p=0.006)$ or $12-14$ years $(p=0.0024)$. The distribution of children with normal weight was significantly different from those in other groups $(p<0.0001)$. 
Table 1. Demographic characteristics of children and adolescents from the CNNHS 2010-2012.

\begin{tabular}{|c|c|c|c|c|}
\hline Variable & Total (N) & Boys (N) & Girls (N) & $p$-Value ${ }^{\text {a }}$ \\
\hline Age group (years) & & & & 0.0150 \\
\hline $6-8$ & 7903 & 4011 & 3892 & \\
\hline 9-11 & 8689 & 4411 & 4278 & \\
\hline $12-14$ & 8794 & 4456 & 4338 & \\
\hline $15-17$ & 7629 & 3843 & 3786 & \\
\hline Region type & & & & 0.2036 \\
\hline Large cities & 6859 & 3455 & 3404 & \\
\hline Small and medium-sized cities & 9729 & 4912 & 4817 & \\
\hline Ordinary rural areas & 10,501 & 5352 & 5149 & \\
\hline Poor rural areas & 5926 & 3002 & 2924 & \\
\hline Ethnicity & & & & 0.0666 \\
\hline Han population & 29,699 & 15,069 & 14,630 & \\
\hline Other population & 3054 & 1498 & 1556 & \\
\hline Latitude & & & & 0.7182 \\
\hline North & 14,943 & 7544 & 7399 & \\
\hline South & 18,072 & 9177 & 8895 & \\
\hline Season & & & & 0.8778 \\
\hline Spring & 2227 & 1135 & 1092 & \\
\hline Summer & 1786 & 899 & 887 & \\
\hline Autumn & 17,801 & 9044 & 8757 & \\
\hline Winter & 10,938 & 5488 & 5450 & \\
\hline BMI classification & & & & $<0.0001$ \\
\hline Underweight & 2884 & 1720 & 1164 & \\
\hline Normal & 24,602 & 11,635 & 12,967 & \\
\hline Overweight & 3307 & 1959 & 1348 & \\
\hline Obesity & 2222 & 1407 & 815 & \\
\hline Income $(\mathrm{RMB}) \mathrm{b}$ & & & & 0.2139 \\
\hline$<10,000$ & 12,607 & 6361 & 6246 & \\
\hline $10,000-19,999$ & 8066 & 4151 & 3915 & \\
\hline $20,000-29,999$ & 2794 & 1453 & 1341 & \\
\hline $30,000-39,999$ & 1206 & 609 & 597 & \\
\hline$\geq 40,000$ & 1055 & 531 & 524 & \\
\hline No response & 6987 & 3440 & 3547 & \\
\hline
\end{tabular}

\subsection{Hemoglobin Concentration of Children and Adolescents}

The hemoglobin levels for all participants in different sub-groups are shown in Table 2. The mean hemoglobin of all participants was $141.2 \pm 15.8 \mathrm{~g} / \mathrm{L}$. Boys had a significantly higher concentration of hemoglobin $(144.0 \pm 16.3 \mathrm{~g} / \mathrm{L})$ than that of girls $(138.0 \pm 14.4 \mathrm{~g} / \mathrm{L}, p<0.0001)$. Significant differences were found in different age groups, region types, latitudes, seasons, BMI levels, and annual family income. Post-hoc analysis showed that participants aged 15-17 years had the highest level of hemoglobin $(p<0.0001)$. Children living in poor rural areas had the lowest levels of hemoglobin $(p<0.0001)$. The hemoglobin level in winter was significantly higher than that in autumn $(p<0.0001)$, spring $(p<0.0001)$, or summer $(p<0.0001)$. Obese children and adolescents had significantly higher hemoglobin levels than those with normal weight $(p<0.0001)$ or those underweight $(p<0.0001)$. Children with annual family incomes between 30,000 and 40,000 RMB had significantly higher hemoglobin levels than those with annual family incomes less than $10,000 \mathrm{RMB}(p=0.0003)$. However, there was no significant difference between the Han population and other populations $(p=0.3462)$. A similar tendency was found in terms of the age group, region type, ethnicity, latitude, season, BMI, and income level in boys and girls. 
Table 2. Hemoglobin concentration of children and adolescents from CNNHS 2010-2012.

\begin{tabular}{|c|c|c|c|c|c|c|}
\hline Variable & Total (g/L) & $p$-Value ${ }^{\mathrm{a}}$ & Boys $(\mathrm{g} / \mathrm{L})$ & $p$-Value ${ }^{b}$ & Girls(g/L) & $p$-Value ${ }^{\mathrm{c}}$ \\
\hline Total & $141.2 \pm 15.8$ & & $144.0 \pm 16.3$ & & $138.0 \pm 14.4$ & \\
\hline Age group (years) & & $<0.0001$ & & $<0.0001$ & & $<0.0001$ \\
\hline $6-8$ & $135.5 \pm 13.4$ & & $135.6 \pm 12.9$ & & $135.4 \pm 13.9$ & \\
\hline $9-11$ & $138.7 \pm 13.5$ & & $138.8 \pm 13.4$ & & $138.7 \pm 13.6$ & \\
\hline $12-14$ & $142.3 \pm 15.8$ & & $145.2 \pm 15.9$ & & $138.9 \pm 14.9$ & \\
\hline $15-17$ & $146.7 \pm 17.1$ & & $153.5 \pm 16.0$ & & $138.9 \pm 14.8$ & \\
\hline Region type & & $<0.0001$ & & $<0.0001$ & & $<0.0001$ \\
\hline Large cities & $142.4 \pm 15.2$ & & $146.0 \pm 15.6$ & & $138.6 \pm 13.8$ & \\
\hline Small and medium-sized cities & $141.0 \pm 16.1$ & & $144.0 \pm 16.6$ & & $137.6 \pm 14.8$ & \\
\hline Ordinary rural areas & $142.0 \pm 15.5$ & & $144.8 \pm 16.2$ & & $138.8 \pm 14.0$ & \\
\hline Poor rural areas & $139.8 \pm 15.5$ & & $141.8 \pm 16.1$ & & $137.4 \pm 14.3$ & \\
\hline Ethnicity & & 0.3462 & & 0.1543 & & 0.6570 \\
\hline Han population & $141.2 \pm 15.7$ & & $144.0 \pm 16.4$ & & $138.0 \pm 14.3$ & \\
\hline Other population & $141.4 \pm 16.0$ & & $144.4 \pm 16.4$ & & $138.2 \pm 14.9$ & \\
\hline Latitude & & $<0.0001$ & & $<0.0001$ & & 0.3373 \\
\hline North & $141.6 \pm 16.0$ & & $144.5 \pm 16.4$ & & $138.2 \pm 14.9$ & \\
\hline South & $140.9 \pm 15.5$ & & $143.6 \pm 16.3$ & & $137.9 \pm 14.0$ & \\
\hline Season & & $<0.0001$ & & $<0.0001$ & & $<0.0001$ \\
\hline Spring & $138.8 \pm 20.6$ & & $141.7 \pm 21.2$ & & $135.5 \pm 19.4$ & \\
\hline Summer & $138.3 \pm 17.7$ & & $140.7 \pm 17.8$ & & $135.4 \pm 17.3$ & \\
\hline Autumn & $140.9 \pm 14.8$ & & $143.5 \pm 15.6$ & & $137.8 \pm 13.1$ & \\
\hline Winter & $142.9 \pm 15.8$ & & $146.0 \pm 16.1$ & & $139.3 \pm 14.6$ & \\
\hline BMI classification & & $<0.0001$ & & $<0.0001$ & & 0.0055 \\
\hline Underweight & $140.2 \pm 16.4$ & & $142.0 \pm 16.7$ & & $137.1 \pm 15.5$ & \\
\hline Normal & $141.2 \pm 15.6$ & & $144.2 \pm 16.3$ & & $138.1 \pm 14.2$ & \\
\hline Overweight & $141.8 \pm 16.3$ & & $144.3 \pm 16.8$ & & $138.0 \pm 14.7$ & \\
\hline Obesity & $142.4 \pm 15.5$ & & $144.5 \pm 15.2$ & & $138.5 \pm 15.2$ & \\
\hline Income $(\mathrm{RMB}) \mathrm{d}$ & & $<0.0001$ & & $<0.0001$ & & 0.1980 \\
\hline$<10,000$ & $140.8 \pm 15.9$ & & $143.2 \pm 16.7$ & & $138.1 \pm 14.5$ & \\
\hline $10,000-20,000$ & $141.3 \pm 15.6$ & & $144.2 \pm 16.0$ & & $138.0 \pm 14.6$ & \\
\hline $20,000-30,000$ & $142.3 \pm 15.9$ & & $145.4 \pm 16.7$ & & $138.6 \pm 13.9$ & \\
\hline $30,000-40,000$ & $142.9 \pm 13.9$ & & $145.4 \pm 14.3$ & & $140.0 \pm 12.7$ & \\
\hline$\geq 40,000$ & $141.9 \pm 14.6$ & & $145.8 \pm 14.6$ & & $137.7 \pm 13.4$ & \\
\hline No response & $141.1 \pm 16.0$ & & $144.6 \pm 16.5$ & & $137.4 \pm 14.6$ & \\
\hline
\end{tabular}

Values are means $\pm \mathrm{SD} ;{ }^{(\mathbf{a}-\mathbf{c})} p$-values for Kruskal-Wallis test; ${ }^{\text {(d) }}$ annual average income per capita; BMI: Body mass index.

\subsection{Anemia Prevalence for Children and Adolescents}

As presented in Table 3, the prevalence of anemia in Chinese children and adolescents in 2010-2012 was $6.6 \%$. Girls had a significantly higher prevalence of anemia $(7.4 \%)$ than boys $(6.0 \%, p<0.001)$. The prevalence of anemia varied among age groups, and the highest levels of anemia were found in adolescents aged 15-17 years (8.6\%). Participants from large cities had the lowest prevalence of anemia $(4.8 \%)$, and those from poor rural areas had the highest prevalence $(9.5 \%)$. With respect to ethnicity, other populations $(7.8 \%)$ had a significantly higher prevalence than the Han population $(6.5 \%, p=0.0096)$. Anemia was more prevalent among participants living in the north than those living in the south $(p=0.0106)$. In spring and summer, the prevalence of anemia was above $10.0 \%$, which was significantly higher than that in autumn and winter (all $p$-values $<0.0001$ ). Obese children and adolescents had the lowest prevalence of anemia (4.3\%). The prevalence of anemia was the highest for participants with an annual income less than 10,000 RMB (7.5\%), and the lowest for those with an annual income above 40,000 RMB. A similar tendency was found in both sexes in terms of their age group, region type, ethnicity, latitude, season, and income level. 
Table 3. Anemia prevalence for children and adolescents from CNNHS 2010-2012.

\begin{tabular}{|c|c|c|c|c|c|c|}
\hline Variable & $\begin{array}{c}\text { Total } \\
(\%(95 \% \mathrm{CI}))\end{array}$ & $p$-Value ${ }^{\text {a }}$ & $\begin{array}{c}\text { Boys } \\
(\%(95 \% \mathrm{CI}))\end{array}$ & $p$-Value ${ }^{\mathrm{b}}$ & $\begin{array}{c}\text { Girls } \\
(\%(95 \% \mathrm{CI}))\end{array}$ & $p$-Value ${ }^{c}$ \\
\hline Total & $\begin{array}{c}6.6 \\
(6.3-6.9)\end{array}$ & & $\begin{array}{c}6.0 \\
(5.6-6.3)\end{array}$ & & $\begin{array}{c}7.4 \\
(6.9-7.8)\end{array}$ & \\
\hline $\begin{array}{l}\text { Age group } \\
\text { (years) }\end{array}$ & & $<0.0001$ & & $<0.0001$ & & $<0.0001$ \\
\hline 6-8 & $\begin{array}{c}5.7 \\
(5.2-6.3)\end{array}$ & & $\begin{array}{c}5.2 \\
(4.5-6.0)\end{array}$ & & $\begin{array}{c}6.3 \\
(5.5-7.1)\end{array}$ & \\
\hline $9-11$ & $\begin{array}{c}4.3 \\
(3.9-4.7)\end{array}$ & & $\begin{array}{c}4.2 \\
(3.5-4.8)\end{array}$ & & $\begin{array}{c}4.5 \\
(3.8-5.1)\end{array}$ & \\
\hline $12-14$ & $\begin{array}{c}7.2 \\
(6.7-7.8)\end{array}$ & & $\begin{array}{c}5.9 \\
(5.1-6.6)\end{array}$ & & $\begin{array}{c}8.9 \\
(8.0-9.8)\end{array}$ & \\
\hline $15-17$ & $\begin{array}{c}8.6 \\
(7.9-9.2)\end{array}$ & & $\begin{array}{c}8.0 \\
(7.1-8.9)\end{array}$ & & $\begin{array}{c}9.2 \\
(8.2-10.2)\end{array}$ & \\
\hline Region type & & $<0.0001$ & & $<0.0001$ & & 0.0003 \\
\hline Large cities & $\begin{array}{c}4.8 \\
(4.3-5.3)\end{array}$ & & $\begin{array}{c}3.4 \\
(2.8-4.0)\end{array}$ & & $\begin{array}{c}6.4 \\
(5.6-7.2)\end{array}$ & \\
\hline Small and medium-sized cities & $\begin{array}{c}6.5 \\
(6.0-7.0)\end{array}$ & & $\begin{array}{c}5.7 \\
(5.1-6.3)\end{array}$ & & $\begin{array}{c}7.5 \\
(6.7-8.2)\end{array}$ & \\
\hline Ordinary rural areas & $\begin{array}{c}5.8 \\
(5.3-6.2)\end{array}$ & & $\begin{array}{c}5.0 \\
(4.4-5.6)\end{array}$ & & $\begin{array}{c}6.6 \\
(5.9-7.3)\end{array}$ & \\
\hline Poor rural areas & $\begin{array}{c}9.5 \\
(8.7-10.2)\end{array}$ & & $\begin{array}{c}9.8 \\
(8.7-10.9)\end{array}$ & & $\begin{array}{c}9.1 \\
(8.0-10.1)\end{array}$ & \\
\hline Ethnicity & & 0.0096 & & 0.5310 & & 0.0033 \\
\hline Han population & $\begin{array}{c}6.5 \\
(6.2-6.8)\end{array}$ & & $\begin{array}{c}5.9 \\
(5.5-6.3)\end{array}$ & & $\begin{array}{c}7.2 \\
(6.7-7.6)\end{array}$ & \\
\hline Other population & $\begin{array}{c}7.8 \\
(6.8-8.8)\end{array}$ & & $\begin{array}{c}6.4 \\
(5.0-7.7)\end{array}$ & & $\begin{array}{c}9.4 \\
(7.8-10.9)\end{array}$ & \\
\hline Latitude & & 0.0106 & & 0.0965 & & 0.0523 \\
\hline North & $\begin{array}{c}7.0 \\
(6.6-7.5)\end{array}$ & & $\begin{array}{c}6.3 \\
(5.7-6.9)\end{array}$ & & $\begin{array}{c}7.8 \\
(7.2-8.5)\end{array}$ & \\
\hline South & $\begin{array}{c}6.2 \\
(5.9-6.6)\end{array}$ & & $\begin{array}{c}5.6 \\
(5.1-6.2)\end{array}$ & & $\begin{array}{c}6.9 \\
(6.3-7.5)\end{array}$ & \\
\hline Season & & $<0.0001$ & & $<0.0001$ & & $<0.0001$ \\
\hline Spring & $\begin{array}{c}15.1 \\
(13.3-16.8)\end{array}$ & & $\begin{array}{c}13.7 \\
(11.4-16.1)\end{array}$ & & $\begin{array}{c}16.6 \\
(14.1-19.2)\end{array}$ & \\
\hline Summer & $\begin{array}{c}13.2 \\
(11.4-14.9)\end{array}$ & & $\begin{array}{c}11.2 \\
(8.9-13.5)\end{array}$ & & $\begin{array}{c}15.5 \\
(12.8-18.1)\end{array}$ & \\
\hline Autumn & $\begin{array}{c}5.4 \\
(5.1-5.8)\end{array}$ & & $\begin{array}{c}5.1 \\
(4.6-5.6)\end{array}$ & & $\begin{array}{l}5.8 \\
(5.3-6.4)\end{array}$ & \\
\hline Winter & $\begin{array}{c}5.8 \\
(5.3-6.3)\end{array}$ & & $\begin{array}{c}5.0 \\
(4.3-5.7)\end{array}$ & & $\begin{array}{c}6.8 \\
(6.0-7.5)\end{array}$ & \\
\hline BMI classification & & $<0.0001$ & & $<0.0001$ & & 0.0793 \\
\hline Underweight & $\begin{array}{c}8.9 \\
(7.8-10.0)\end{array}$ & & $\begin{array}{c}8.6 \\
(7.2-10.0)\end{array}$ & & $\begin{array}{c}9.4 \\
(7.6-11.2)\end{array}$ & \\
\hline Normal & $\begin{array}{c}6.6 \\
(6.2-6.9)\end{array}$ & & $\begin{array}{c}5.9 \\
(5.5-6.4)\end{array}$ & & $\begin{array}{c}7.2 \\
(6.7-7.7)\end{array}$ & \\
\hline Overweight & $\begin{array}{c}6.3 \\
(5.4-7.3)\end{array}$ & & $\begin{array}{c}5.6 \\
(4.4-6.7)\end{array}$ & & $\begin{array}{c}7.5 \\
(5.9-9.1)\end{array}$ & \\
\hline Obesity & $\begin{array}{c}4.3 \\
(3.4-5.3)\end{array}$ & & $\begin{array}{c}3.2 \\
(2.2-4.2)\end{array}$ & & $\begin{array}{c}6.4 \\
(4.5-8.3)\end{array}$ & \\
\hline $\begin{array}{l}\text { Income } \\
(\text { RMB })^{d}\end{array}$ & & $<0.0001$ & & $<0.0001$ & & 0.0415 \\
\hline$<10,000$ & $\begin{array}{c}7.5 \\
(7.0-7.9)\end{array}$ & & $\begin{array}{c}7.4 \\
(6.7-8.0)\end{array}$ & & $\begin{array}{c}7.6 \\
(6.9-8.3)\end{array}$ & \\
\hline $10,000-20,000$ & $\begin{array}{c}6.6 \\
(6.0-7.2)\end{array}$ & & $\begin{array}{c}5.4 \\
(4.6-6.1)\end{array}$ & & $\begin{array}{c}8.0 \\
(7.0-8.9)\end{array}$ & \\
\hline $20,000-30,000$ & $\begin{array}{c}6.0 \\
(5.1-7.0)\end{array}$ & & $\begin{array}{c}5.7 \\
(4.3-7.0)\end{array}$ & & $\begin{array}{c}6.5 \\
(5.0-8.0)\end{array}$ & \\
\hline $30,000-40,000$ & $\begin{array}{c}4.0 \\
(2.7-5.2)\end{array}$ & & $\begin{array}{c}3.5 \\
(1.8-5.2)\end{array}$ & & $\begin{array}{c}4.5 \\
(2.7-6.3)\end{array}$ & \\
\hline$\geq 40,000$ & $\begin{array}{c}3.6 \\
(2.3-4.9)\end{array}$ & & $\begin{array}{c}2.1 \\
(0.6-3.5)\end{array}$ & & $\begin{array}{c}5.3 \\
(3.2-7.5)\end{array}$ & \\
\hline No response & $\begin{array}{c}6.2 \\
(5.6-6.8)\end{array}$ & & $\begin{array}{c}5.1 \\
(4.3-6.0)\end{array}$ & & $\begin{array}{c}7.4 \\
(6.4-8.3)\end{array}$ & \\
\hline
\end{tabular}

Values are presented as \% with $95 \% \mathrm{CI}$ in parentheses; ${ }^{\text {(a-c) }} p$-value for the Rao-Scott test; ${ }^{(\mathbf{d})}$ annual average income per capita; BMI: Body mass index.

3.4. Comparison of Anemia Status for Children and Adolescents between CNNHS 2010-2012 and CNNHS2002

As presented in Table 4, hemoglobin concentrations markedly increased over the decade, from 135.2 $\pm 13.9 \mathrm{~g} / \mathrm{L}$ in 2002 to $141.2 \pm 15.8 \mathrm{~g} / \mathrm{L}$ in 2010-2012 $(p<0.001)$. This remarkable upward trend was similar 
in each sub-group, including different age groups, sexes, ethnicities, and region types. In addition, the prevalence of anemia significantly decreased over the same time span, from $12.6 \%$ in 2002 to $6.6 \%$ in 2010-2012 $(p<0.001)$. This clear downward trend was found in each sub-group, including different age groups, sexes, ethnicities, and region types. Among them, the prevalence of anemia in the four different age groups, minorities, and rural areas declined by more than half.

Table 4. Comparison of hemoglobin concentration and anemia prevalence for children and adolescents between CNNHS 2010-2012 and CNNHS 2002.

\begin{tabular}{|c|c|c|c|c|c|c|c|c|c|}
\hline \multirow{2}{*}{ Variable } & \multicolumn{3}{|c|}{$\mathbf{N}$} & \multicolumn{3}{|c|}{$\mathrm{Hb}(\mathrm{g} / \mathrm{L})^{\mathrm{a}}$} & \multicolumn{3}{|c|}{ Prevalence $(\%(95 \% \mathrm{CI}))^{b}$} \\
\hline & 2002 & 2010-2012 & $p$-Value ${ }^{\mathrm{c}}$ & 2002 & 2010-2012 & $p$-Value ${ }^{\mathrm{d}}$ & 2002 & 2010-2012 & $p$-Value ${ }^{\mathrm{e}}$ \\
\hline Total & 48,610 & 33,015 & \multirow{3}{*}{$<0.0001$} & $135.2 \pm 13.9$ & $141.2 \pm 15.8$ & $<0.0001$ & $\begin{array}{c}12.6 \\
(12.3-12.9)\end{array}$ & $\begin{array}{c}6.6 \\
(6.3-6.9)\end{array}$ & $<0.0001$ \\
\hline Normal & 42,472 & 30,937 & & $138.4 \pm 11.3$ & $143.4 \pm 13.4$ & $<0.0001$ & - & - & \\
\hline Anemia & 6138 & 2078 & & $113.2 \pm 9.7$ & $110.1 \pm 13.1$ & $<0.0001$ & $\begin{array}{c}12.6 \\
(12.3-12.9)\end{array}$ & $\begin{array}{c}6.6 \\
(6.3-6.9)\end{array}$ & $<0.0001$ \\
\hline \multicolumn{10}{|c|}{ Age group (years) } \\
\hline $6-8$ & 14,678 & 7903 & \multirow{4}{*}{$<0.0001$} & $132.2 \pm 12.8$ & $135.5 \pm 13.4$ & $<0.0001$ & $\begin{array}{c}11.8 \\
(11.3-12.3)\end{array}$ & $\begin{array}{c}5.7 \\
(5.2-6.3)\end{array}$ & $<0.0001$ \\
\hline $9-11$ & 16,020 & 8689 & & $134.5 \pm 12.8$ & $138.7 \pm 13.5$ & $<0.0001$ & $\begin{array}{c}9.6 \\
(9.1-10.1)\end{array}$ & $\begin{array}{c}4.3 \\
(3.9-4.7)\end{array}$ & $<0.0001$ \\
\hline $12-14$ & 12,174 & 8794 & & $137.0 \pm 14.2$ & $142.3 \pm 15.8$ & $<0.0001$ & $\begin{array}{c}14.8 \\
(14.2-15.4)\end{array}$ & $\begin{array}{c}7.2 \\
(6.7-7.8)\end{array}$ & $<0.0001$ \\
\hline $15-17$ & 5738 & 7629 & & $140.9 \pm 16.3$ & $146.7 \pm 17.1$ & $<0.0001$ & $\begin{array}{c}18.5 \\
(17.5-19.5)\end{array}$ & $\begin{array}{c}8.6 \\
(7.9-9.2)\end{array}$ & $<0.0001$ \\
\hline \multicolumn{10}{|l|}{ Sex } \\
\hline Boys & 25,133 & 16,721 & \multirow[t]{2}{*}{0.003} & $136.3 \pm 14.5$ & $144.0 \pm 16.3$ & $<0.0001$ & $\begin{array}{c}12.1 \\
(11.7-12.5)\end{array}$ & $\begin{array}{c}6.0 \\
(5.6-6.3)\end{array}$ & $<0.0001$ \\
\hline Girls & 23,476 & 16,294 & & $134.0 \pm 13.3$ & $138.0 \pm 14.4$ & $<0.0001$ & $\begin{array}{c}13.1 \\
(12.7-13.6)\end{array}$ & $\begin{array}{c}7.4 \\
(6.9-7.8)\end{array}$ & $<0.0001$ \\
\hline \multicolumn{10}{|l|}{ Ethnicity } \\
\hline Han & 42,356 & 29,699 & \multirow{2}{*}{$<0.0001$} & $135.0 \pm 13.7$ & $141.2 \pm 15.7$ & $<0.0001$ & $\begin{array}{c}12.1 \\
(11.7-12.4)\end{array}$ & $\begin{array}{c}6.5 \\
(6.2-6.8)\end{array}$ & $<0.0001$ \\
\hline $\begin{array}{c}\text { Other } \\
\text { population }\end{array}$ & 6254 & 3054 & & $136.7 \pm 15.2$ & $141.4 \pm 16.0$ & $<0.0001$ & $\begin{array}{c}16.5 \\
(15.5-17.4)\end{array}$ & $\begin{array}{c}7.8 \\
(6.8-8.8)\end{array}$ & 0.0001 \\
\hline \multicolumn{10}{|c|}{ Region type } \\
\hline Urban & 21,468 & 16,588 & \multirow[t]{2}{*}{$<0.0001$} & $135.7 \pm 13.5$ & $141.1 \pm 16.0$ & $<0.0001$ & $\begin{array}{c}9.5 \\
(9.1-9.8)\end{array}$ & $\begin{array}{c}6.3 \\
(5.9-6.7)\end{array}$ & $<0.0001$ \\
\hline Rural & 27,142 & 16,427 & & $134.8 \pm 14.3$ & $141.3 \pm 15.5$ & $<0.0001$ & $\begin{array}{c}15.1 \\
(14.7-15.6)\end{array}$ & $\begin{array}{c}6.9 \\
(6.5-7.3)\end{array}$ & $<0.0001$ \\
\hline
\end{tabular}

(a) Values are means $\pm \mathrm{SD}$; ${ }^{\text {(b) }}$ values are presented as \% $(95 \% \mathrm{CI})$; ${ }^{\text {(c) }} p$-value for the Rao-Scott; ${ }^{(\mathrm{d})} p$-value for Kruskal-Wallis test; (e) $p$-value for the Rao-Scott test.

\subsection{Association between Anemia and Relative Variables}

Factors including sex, age, region type, ethnicity, latitude, season, BMI level, and annual income were examined using a multi-variable logistic regression analysis for participants in CNNHS 2010-2012. The total number of participants was 32,714. As shown in Table 5, anemia was significantly associated with the female sex (OR $=1.275, p<0.0001$, relative to boys) and ages of 6-8 years and 12-17 years (ages 6-8 years, $\mathrm{OR}=1.309, p=0.0175$; ages $12-14$ years, $\mathrm{OR}=1.676, p=0.0007$; ages $15-17$ years, $\mathrm{OR}=2.065, p<0.0001$; relative to those aged 9-11 years). Participants from ordinary rural areas or poor rural areas displayed an increased risk of anemia; the ORs were $1.205(p=0.0009)$ and 2.182 $(p<0.0001)$, respectively. Taking autumn as the reference standard, other seasons' risks of anemia were increased, and the OR was 3.155 for spring $(p<0.0001), 2.819$ for summer $(p<0.0001)$ and 1.091 for winter $(p<0.0001)$. An increased risk of anemia was observed in underweight children $(\mathrm{OR}=1.741$, $p<0.0001$, relative to a BMI level above $28 \mathrm{~kg} / \mathrm{m}^{2}$ ). Participants whose annual income was less than 20,000 RMB had an increased risk of anemia; with respect to an income level of more than 40,000 RMB, the OR was 1.704 for less than 10,000 RMB ( $p=0.0001)$ and 1.608 for 10,000-19,999 RMB $(p=0.0099)$. However, no significant difference was observed in the risk of anemia according to ethnicity or latitude. 
Table 5. Determinants of anemia among Chinese children and adolescents from CNNHS 2010-2012.

\begin{tabular}{|c|c|c|}
\hline Variable & OR $(95 \% \mathrm{CI})^{\mathrm{a}}$ & $p$-Value ${ }^{b}$ \\
\hline \multicolumn{3}{|l|}{ Sex } \\
\hline Boys & ref & \\
\hline Girls & $1.275(1.155,1.407)$ & $<0.0001$ \\
\hline \multicolumn{3}{|l|}{ Age group (years) } \\
\hline $9-11$ & ref & \\
\hline $6-8$ & $1.309(1.124,1.525)$ & 0.0175 \\
\hline $12-14$ & $1.676(1.453,1.932)$ & 0.0007 \\
\hline 15-17 & $2.065(1.794,2.376)$ & $<0.0001$ \\
\hline \multicolumn{3}{|l|}{ Region type } \\
\hline Large cities & ref & \\
\hline Small and medium-sized cities & $1.388(1.209,1.593)$ & 0.9167 \\
\hline Ordinary rural areas & $1.205(1.040,1.396)$ & 0.0009 \\
\hline Poor rural areas & $2.182(1.869,2.548)$ & $<0.0001$ \\
\hline \multicolumn{3}{|l|}{ Ethnicity } \\
\hline Han & ref & \\
\hline Other population & $0.894(0.762,1.048)$ & 0.1667 \\
\hline \multicolumn{3}{|l|}{ Latitude } \\
\hline South & ref & \\
\hline North & $1.011(0.918,1.114)$ & 0.8164 \\
\hline \multicolumn{3}{|l|}{ Season } \\
\hline Autumn & ref & \\
\hline Spring & $3.155(2.711,3.671)$ & $<0.0001$ \\
\hline Summer & $2.819(2.387,3.33)$ & $<0.0001$ \\
\hline Winter & $1.091(0.966,1.231)$ & $<0.0001$ \\
\hline \multicolumn{3}{|l|}{ BMI classification } \\
\hline Obesity & ref & \\
\hline Underweight & $1.741(1.324,2.289)$ & $<0.0001$ \\
\hline Normal & $1.302(1.026,1.652)$ & 0.6464 \\
\hline Overweight & $1.380(1.043,1.824)$ & 0.6019 \\
\hline \multicolumn{3}{|l|}{ Income $(\mathrm{RMB})^{\mathrm{c}}$} \\
\hline$\geq 40,000$ & ref & \\
\hline$<10,000$ & $1.704(1.168,2.485)$ & 0.0001 \\
\hline $10,000-19,999$ & $1.608(1.098,2.354)$ & 0.0099 \\
\hline $20,000-29,999$ & $1.589(1.056,2.391)$ & 0.0875 \\
\hline $30,000-39,999$ & $1.058(0.647,1.731)$ & 0.0737 \\
\hline No response & $1.425(0.970,2.093)$ & 0.5374 \\
\hline
\end{tabular}

(a) Values are presented as $\%(95 \% \mathrm{CI}){ }^{\text {(b) }} p$-value for multi-variable logistic regression analysis; ${ }^{\text {(c) }}$ annual average income per capita; BMI: Body mass index.

\section{Discussion}

In this study, we showed that the mean hemoglobin concentration of Chinese children and adolescents in CNNHS 2010-2012 was $141.2 \mathrm{~g} / \mathrm{L}$, which was a marked improvement compared with CNNHS 2002 findings. A significant decrease in the prevalence of anemia was observed from $12.6 \%$ in CNNHS 2002 to 6.6\% in CNNHS 2010-2012. On the whole, the prevalence of anemia in Chinese children and adolescents represented only a mild public health problem during 2010-2012 according to the WHO criteria [23]. The improvement was in accordance with a recent study conducted by Song et al., who assessed the trend in sex disparity in hemoglobin concentration and the prevalence of anemia among Chinese school-aged children from 1995 to 2010 [24]. They found that the mean hemoglobin concentration increased among Chinese school-aged children between 1995 and 2010, from $132.7 \mathrm{~g} / \mathrm{L}$ to $138.3 \mathrm{~g} / \mathrm{L}$ in boys, and from $127.7 \mathrm{~g} / \mathrm{L}$ to $132.3 \mathrm{~g} / \mathrm{L}$ in girls. The prevalence of anemia decreased from $18.8 \%$ in 1995 to $9.9 \%$ in 2010 . However, such data were obtained from a different survey, the Chinese National Survey on Students Constitution and Health (CNSSCH), whose participants were all Han children aged 7, 9, 12, 14, and 17 years old, and samples of capillary blood were collected from 
the fingertip of each child. These differences might have produced the difference in the hemoglobin concentration values and the prevalence of anemia between CNNHS 2010-2012 and CNSSCH 2010.

We found that hemoglobin concentration and the prevalence of anemia varied with sex, age group, region type, season, BMI level, and annual income. The mean hemoglobin concentration of boys was significantly higher than that of girls, so the prevalence of anemia for boys was significantly lower than that for girls. Girls lose blood during menstruation when they enter puberty and thus are more vulnerable to anemia than boys. The difference between sexes in hemoglobin concentration and the prevalence of anemia was also observed in previous studies [11,24,25]. However, some studies reported no significant difference in the mean hemoglobin concentration between boys and girls, and the reason for this could be the different sample sizes and subjects [26,27].

Compared with those living in cities or ordinary rural areas in CNNHS 2010-2012, children and adolescents living in poor rural areas showed the highest prevalence and were at a greater risk of anemia. Although the Chinese economy has grown rapidly in both urban and rural areas [14], living conditions in urban areas remain better than those in rural areas $[28,29]$. Thus, the nutritional status of people in cities is often better than those in rural areas. Compared with the Han population, ethnic minorities had a higher prevalence of anemia. In China, ethnic minorities often live in remote regions, and their living standards are not as good as those of the Han population. Song et al. found that the prevalence of anemia was different among students of different ethnic minorities [12]. The prevalence of anemia for Hui ethnic students was a moderate public health problem according to the WHO criteria [12]. Although the effects of seasonal changes on anemia have rarely been reported, they may be an important factor. The results of the current study indicate that the highest prevalence of anemia occurs in spring, followed by summer. Ronnenberg et al. reported that distinct seasonal trends were observed in the prevalence of moderate anemia for Chinese women of a childbearing age [29,30]. The hemoglobin concentrations of these women were significantly lower in summer than those in other seasons [30]. Chinese people often have a strong sense of strengthening their resistance to a cold winter by consuming more red meat in autumn, whereas in spring and summer, people often have less of an appetite because of the hot weather.

Overweight and obese children had a significantly lower prevalence of anemia than those with normal or low body weight. This is in accordance with reports on Chinese people by He et al. and Qin et al. [31,32]. Bentley et al. also found that overweight women were at a lower risk of anemia than those with a normal or low BMI in India, which could be due to a better dietary nutritional intake in overweight or obese people [33]. However, some studies found that obesity is significantly associated with iron deficiency, and could be caused by obesity-related inflammation and/or related comorbidities $[34,35]$. The disparity among those findings might be due to the different dietary patterns in different regions. The relationship between BMI and anemia needs to be further researched. Family income also had an impact on anemia. The highest prevalence of and vulnerability to anemia was observed in children whose family income was less than 10,000 RMB per year. This could be explained by the fact that their parents may have less ability and awareness to improve their children's nutrition due to their poor socioeconomic and household conditions. Previous studies demonstrated associations between anemia and indicators of poor socioeconomic conditions in China [36] and in other developing countries, such as parts of Africa [37,38]. Li et al. found a negative correlation between household wealth and the prevalence of anemia in Central and Eastern China [11]. Ngnie-Teta et al. [37] and Custodio et al. [38] found that children who lived in households with lower living standards or lower socio-educational levels were at greater risk of anemia.

However, considerable improvement has occurred in terms of hemoglobin concentration and the prevalence of anemia in different age groups, sexes, ethnicities, and regions in Chinese children and adolescents since 2002. Efforts have been made to decrease the prevalence of anemia in China. Firstly, some specific nutritional policies implemented by the government have played an important role in alleviating anemia, such as The Standard Amount of Nutritional Supply for Student Lunch issued by the Ministry of Health in 1998 [39], and The Nutrition Improvement Program for Rural 
Compulsory Education Students issued by the State Council in October 2011 [40]. As the population at the highest risk of anemia, rural school-aged children, especially rural girls, may have benefitted from these policies. Secondly, public health strategies contributed to preventing and improving resistance to anemia, such as food fortification, micronutrient supplementation, nutritional and public health awareness enhancement, and the improvement in dietary diversity [41]. Thirdly, with the growing economy, the living standards of Chinese citizens have been improved, providing more opportunity for people to consume more iron-rich foods.

There were some limitations to this study. Firstly, the iron status of children was not examined; thus, it is not possible to speculate about the causal relationship between iron and anemia in children and adolescents. The survey did not include some causes of improvement in anemia rates, such as iron supplements or other food fortifications. Lastly, this survey did not consider that shifting the nutrient status for a whole population may lead to some people being exposed to excess levels of a nutrient. Further surveys need to pay more attention to these aspects to avoid these limitations.

\section{Conclusions}

In summary, hemoglobin levels and prevalence of anemia have improved among Chinese children and adolescents between 2002 and 2012. However, the prevalence of anemia remained relatively high for girls, 15-17-year-old youths, rural areas, and in households with a low income. Children and adolescents aged 6-17 years are at an important stage of physical and mental development. Further research is required to understand the determinants of iron status, which could then lead to strategies to alleviate iron deficiency for Chinese children and adolescents, especially for girls, those living in rural areas, underweight individuals, and those with a low family income.

Author Contributions: Conceptualization, X.Y.; Methodology, X.Y. and J.P.; Investigation, J.C., D.M., W.L., R.W., Y.Y.; Data Curation, J.W., Y.H and M.L.; Writing-Original Draft Preparation, J.W.; Writing-Review \& Editing, L.Y.; Supervision, J.P.; Project Administration, L.Y. and X.Y.

Funding: This research was funded by the Special Fund for Health-Scientific Research in the Public Interest (No. 20120212) from the National Health Commission of the People's Republic of China, and The APC was funded by the National Institute for Nutrition and Health, Chinese Center for Disease Control and Prevention.

Acknowledgments: This research was funded by the Special Fund for Health-Scientific Research in the Public Interest (No. 20120212) from the National Health Commission of the People's Republic of China. We thank all the participants in our study and the staff working for the CHNNS 2010-2012 and CHNNS 2002.

Conflicts of Interest: The authors declare no conflict of interest.

\section{References}

1. Stevens, G.A.; Finucane, M.M.; De-Regil, L.M.; Paciorek, C.J.; Flaxman, S.R.; Branca, F.; Peña-Rosas, J.P.; Bhutta, Z.A.; Ezzati, M. Nutrition Impact Model Study Group (Anaemia). Global, regional, and national trends in haemoglobin concentration and prevalence of total and severe anaemia in children and pregnant and non-pregnant women for 1995-2011: A systematic analysis of population-representative data. Lancet Glob. Health 2013, 1, e16-e25. [PubMed]

2. Horton, S.; Levin, C. Commentary on "evidence that iron defciency anemia causes reduced work capacity". J. Nutr. 2001, 131, 691S-696S. [CrossRef] [PubMed]

3. Crawley, J. Reducing the burden of anemia in infants and young children in malaria- endemic countries of Africa: From evidence to action. Am. J. Trop. Med. Hyg. 2004, 71, 25-34. [CrossRef] [PubMed]

4. Balarajan, Y.; Ramakrishnan, U.; Ozaltin, E.; Shankar, A.H.; Subramanian, S.V. Anaemia in low-income and middle-income countries. Lancet 2011, 378, 2123-2135. [CrossRef]

5. Kassebaum, N.J.; Jasrasaria, R.; Naghavi, M.; Wulf, S.K.; Johns, N.; Lozano, R.; Regan, M.; Weatherall, D.; Chou, D.P.; Eisele, T.P.; et al. A systematic analysis of global anemia burden from 1990 to 2010. Blood 2014, 123, 615-624. [CrossRef] [PubMed]

6. World Health Organization (WHO). The Global Prevalence of Anaemia in 2011. Available online: http://apps.who.int/iris/bitstream/handle/10665/177094/9789241564960_eng.pdf?sequence=1 (accessed on 12 January 2018). 
7. World Health Organization (WHO). Iron Deficiency Anaemia: Assessment, Prevention, and Control. Available online: https:/www.who.int/nutrition/publications/micronutrients//anaemia_iron_deficiency/ WHO_NHD_01.3/en/ (accessed on 13 January 2018).

8. GBD 2013 Mortality and Causes of Death Collaborators. Global, regional, and national age-sex specific all-cause and cause-specific mortality for 240 causes of death, 1990-2013: A systematic analysis for the global burden of disease study 2013. Lancet 2015, 385, 117-171. [CrossRef]

9. World Health Organization (WHO). Anaemia. Available online: http://www.who.int/topics/anaemia/en/ (accessed on 30 December 2017).

10. Luo, R.; Zhang, L.; Liu, C.; Zhao, Q.; Shi, Y.; Miller, G.; Yu, E.; Sharbono, B.; Medina, A.; Rozelle, S.; et al. Anaemia among students of rural China's elementary schools: Prevalence and correlates in Ningxia and Qinghai's poor counties. J. Health Popul. Nutr. 2011, 29, 471-485. [CrossRef] [PubMed]

11. Li, L.; Luo, R.; Medina, A.; Rozelle, S. The prevalence of anemia in central and eastern China: Evidencefrom the China health and nutrition survey. Southeast Asian J. Trop. Med. Public Health 2015, 46, 306-321. [PubMed]

12. Song, Y.; Zhang, B.; Hu, P.; Ma, J. Prevalence of anemia and its association with nutritional status among Chinese students of ethnic minorities in 2010. J. Peking Univ. (Health Sci.) 2016, 48, 429-435.

13. Yang, X.G.; Zai, F.Y. Physical and Nutritional Status of Residents; People's Health Publishing Press: Beijing, China, 2006; pp. 15-30.

14. National Bureau of Statistics of China. Gross Domestic Product (GDP) during past 20 Years. Available online: http://data.stats.gov.cn/easyquery.htm?cn=C01 (accessed on 30 November 2016.).

15. Chang, J.L.; Wang, Y. Chinese Nutrition and Health Surveillance-the Comprehensive Report between 2010-2013; Peking University Medical Press: Beijing, China, 2016; pp. 18-30.

16. Li, M.; Hu, Y.; Mao, D.; Wang, R.; Chen, J.; Li, W.; Yang, X.; Piao, J.; Yang, L. Prevalence of anemia among Chinese rural residents. Nutrients 2017, 9, 192. [CrossRef] [PubMed]

17. Hu, Y.; Chen, J.; Wang, R.; Li, M.; Yun, C.; Li, W.; Yang, Y.; Piao, J.; Yang, X.; Yang, L. Vitamin D nutritional status and its related factors for Chinese children and adolescents in 2010-2012. Nutrients 2017, 9, 1024. [CrossRef] [PubMed]

18. The State Council of the People's Republic of China. Outline of Poverty Alleviation and Development in China's Rural Areas (2001-2010). Chin. Law Gov. 2011, 44, 10-20. [CrossRef]

19. Zhao, L.; Ma, G.; Piao, J.; Zhang, J.; Yu, D.; He, Y.; Huo, J.; Hu, X.; Yang, Z.; Yang, X. Scheme of the 2010-2012 Chinese nutrition and health surveillance. Zhonghua Yu Fang Yi Xue Za Zhi 2016, 50, 204-207.

20. Zwart, A.; van Assendelft, O.W.; Bull, B.S.; England, J.M.; Lewis, S.M.; Zijlstra, W.G. Recommendations for reference method for haemoglobinmetry in human blood (ISCH standard 1995) and specifications for international haemiglobinocyanide standard (4th edition). J. Clin. Pathol. 1996, 49, 271-274. [CrossRef] [PubMed]

21. Chinese Nutrition Society. Dietary Guidelines for Chinese School-aged Children; People's Health Publishing Press: Beijing, China, 2016; pp. 10-21.

22. The National Bureau of Statistics of China. Available online: http://data.stats.gov.cn/easyquery.htm? $\mathrm{cn}=$ $\mathrm{C} 01 \& \mathrm{zb}=\mathrm{A} 0301 \& \mathrm{sj}=2009$ (accessed on 15 January 2018).

23. World Health Organization (WHO). The Management of Nutrition in Major emergencies. Available online: http://www.who.int/nutrition/publications/emergencies/9241545208/en/ (accessed on 30 December 2017).

24. Song, Y.; Wang, H.J.; Dong, B.; Wang, Z.; Ma, J.; Agardh, A. National trends in haemoglobin. concentration and prevalence of anemia among Chinese school-aged children, 1995-2010. J. Pediatr. 2017, 183, 164-169. [CrossRef]

25. Egbi, G.; Steiner-Asiedu, M.; Kwesi, F.S.; Ayi, I.; Ofosu, W.; Setorglo, J.; Klobodu, S.S.; Armar-Klemesu, M. Anaemia among school children older than five years in the Volta Region of Ghana. Pan Afr. Med. J. 2014, 1 (Suppl. S17), 10. [CrossRef]

26. Achouri, I.; Aboussaleh, Y.; Sbaibi, R.; Ahami, A.; El Hioui, M. Prevalence of iron deficiency anaemia among school children in Kenitra, Northwest of Morocco. Pak. J. Biol Sci. 2015, 18, 191-195. [CrossRef] [PubMed]

27. Le Nguyen, B.K.; Le Thi, H.; Thuy, N.T.; Huu, C.N.; Do, T.T.; Deurenberg, P.; Khouw, I. Double burden of undernutrition and overnutrition in Vietnam in 2011: Results of the SEANUTS study in 0.5-11-year-old children. Br. J. Nutr. 2013, 110 (Suppl. S3), S45-S56. [CrossRef] [PubMed]

28. National Bureau of Statistics of China. 10-1 Basic Statistics on People's Living Conditions. Available online: http://www.stats.gov.cn/tjsj/ndsj/2011/html/J1001e.htm (accessed on 20 November 2017). 
29. National Bureau of Statistics of China. 10-2 per Capita Annual Income and Engel's Coefficient of Urban and Rural Households. Available online: http://www.stats.gov.cn/tjsj/ndsj/2011/html/J1002e.htm (accessed on 30 December 2017).

30. Ronnenberg, A.G.; Goldman, M.B.; Aitken, I.W.; Xu, X. Anemia and deficiencies of folate and vitamin B-6 are common and vary with season in Chinese women of childbearing age. J. Nutr. 2000, 130, 2703-2710. [CrossRef]

31. He, B.; Chen, Y.; Zhu, Y.; Mai, J.; Jing, J.; Bao, F.; Yu, M.; Li, X.; Jin, Y. Study on the association between BMI and anemia in 7 to 12 years old children in Guangzhou,2010. Chin. J. Prev Med. 2014, 48, 1115-1117. (In Chinese)

32. Qin, Y.; Melse-Boonstra, A.; Pan, X.; Yuan, B.; Dai, Y.; Zhao, J.; Zimmermann, M.B.; Kok, F.J.; Zhou, M.; Shi, Z. Anemia in relation to body mass index and waist circumference among Chinese women. Nutr. J. 2013, 12, 10. [CrossRef] [PubMed]

33. Bentley, M.E.; Griffiths, P.L. The burden of anemia among women in India. Eur. J. Clin. Nutr. 2003, 57, 52-60. [CrossRef] [PubMed]

34. Zhao, L.; Zhang, X.; Shen, Y.; Fang, X.; Wang, Y.; Wang, F. Obesity and iron deficiency: A quantitative meta-analysis. Obes. Rev. 2015, 16, 108-1093. [CrossRef] [PubMed]

35. Jones, A.D.; Zhao, G.; Jiang, Y.P.; Zhou, M.; Xu, G.; Kaciroti, N.; Zhang, Z.; Lozoff, B. Maternal obesity during pregnancy is negatively associated with maternal and neonatal iron status. Eur. J. Clin. Nutr. 2016, 70, 918-924. [CrossRef]

36. Luo, R.; Kleiman-Weiner, M.; Rozelle, S.; Zhang, L.; Liu, C.; Sharbono, B.; Shi, Y.; Yue, A.; Martorell, R.; Lee, M. Anemia in rural China's elementary schools: Prevalence and correlates in Shaanxi province's poor counties. Ecol. Food Nutr. 2010, 49, 357-372. [CrossRef] [PubMed]

37. Ngnie-Teta, I.; Receveur, O.; Kuate-Defo, B. Risk factors for moderate to severe anemia among children in Benin and Mali: Insights froma multilevel analysis. Food Nutr. Bull. 2007, 28, 76-89. [CrossRef] [PubMed]

38. Custodio, E.; Descalzo, M.A.; Roche, J.; Sánchez, I.; Molina, L.; Lwanga, M.; Bernis, C.; Villamor, E.; Baylin, A. Nutritional status and its correlates in Equatorial Guinean preschool children: Results from a nationally representative survey. Food Nutr. Bull. 2008, 29, 49-58. [CrossRef] [PubMed]

39. Amount of Nutritional Supply for Student Lunch; WS/T100-1998; People's Republic of China, Health Industry Standard: Beijing, China, 1998; pp. 1-6.

40. The Central People's Government of the People's Republic of China. The inform of the State Council on the Nutrition Improvement Program for Rural Compulsory Education Students. Available online: http: //www.moe.gov.cn/jyb_xwfb/s6052/moe_838/201110/t20111026_125887.html (accessed on 30 December 2017).

41. Chen, J.; Zhao, X.; Zhang, X.; Yin, S.; Piao, J.; Huo, J.; Yu, B.; Qu, N.; Lu, Q.; Wang, S.; et al. Studies on the effectiveness of NaFeEDTA-fortified soysauce in controlling iron deficiency: A population-based intervention trial. Food Nutr. Bull. 2005, 26, 177-186. [CrossRef] [PubMed]

(C) 2019 by the authors. Licensee MDPI, Basel, Switzerland. This article is an open access article distributed under the terms and conditions of the Creative Commons Attribution (CC BY) license (http://creativecommons.org/licenses/by/4.0/). 